\title{
A Comprehensive Review of Pharmaceutical Care in Type 2 Diabetes Patients; Keeping Developing Countries in View
}

\author{
B. O. Ogbonna ${ }^{1 *}$ and A. C. Oparah ${ }^{2}$ \\ 'Department of Clinical Pharmacy and Pharmacy Management, Faculty of Pharmaceutical Sciences, \\ Nnamdi Azikiwe University, Awka, Nigeria; bo.ogbonna@unizik.edu.ng \\ 2Department of Clinical Pharmacy and Pharmacy Practice, Faculty of Pharmacy, \\ University of Benin, Benin City, Nigeria
}

\begin{abstract}
Pharmaceutical Care (PC) represents a frontier in disease state management. It is a departure from the traditional drug dispensing of the past to comprehensive and holistic focus on patient disease management and outcomes of pharmacotherapy while systematically and actively identifying potential and actual problems in pharmacotherapy. This narrative review assessed the benefits of pharmaceutical care in promoting patient care and disease management. The study utilized synthesis of materials obtained retrospectively from officially reorganized databases. Some of the articles were obtained from officially reorganized documents. PC is patient oriented and empowers patients to actively participate in their treatment process. Pharmacist's device means of monitoring pharmacotherapy outcomes and systematically track problems of pharmacotherapy while taking responsibility for their actions. PC improves patients' outcomes, reduce cost, and promote patients quality of life.
\end{abstract}

Keywords: Patient Care, Pharmacist, Pharmaceutical Care, Pharmacotherapy

\section{Introduction}

\subsection{Overview of Pharmaceutical Care}

Pharmacy as a profession has passed through the processes of transition since inception. These processes of change in terms of philosophy and practice are usually in response to meet the ever-changing social needs of the profession of pharmacy. Modern pharmacy practice has evolved from a product-oriented practice to patient-oriented practice. In the product-oriented practice, the pharmacist primarily supervises medication distribution and counseling of patients. The patient-oriented practice involves a more expanded and team-based clinical role, providing patient centered medication therapy management, health improvement, and disease prevention services. Pharmaceutical care is the responsible provision of pharmacotherapy designed to achieve definite outcomes that improve a patient's quality of life $\mathrm{f}^{1,2}$.
The outcomes of PC are curing a disease, reducing or eliminating symptoms, and slowing or arresting a disease process, and elimination of symptoms before they arise. The launch of pharmaceutical care as the "responsible provision of drug therapy for the purpose of achieving definite outcomes that improve a patient's quality of life" was a major landmark in the history of pharmacy practice. In reality, the pharmaceutical care model was an incremental step forward, rather than a revolutionary leap. This was because the foundations of pharmaceutical care such as the determination of drug needs for a given individual patients and the provision of the drug required, has been defined. Other reasons are that the necessary services to assure optimal safe and effective therapy had already been established ${ }^{3}$.After over two decades into the advent of PC, with ever increasing values it adds to patient care process and evidence based benefits to health care elucidated by scholarly studies, PC care has remained

*Author for correspondence 
at the developmental stage in most African countries. The need to embrace the positive dynamic trend, which will be invaluable in resource-limited settings, has necessitated this study. This review assessed the benefits of pharmaceutical care in promoting patient care and disease management.

\section{Methods}

Articles were sourced from PUBMED, MEDLINE, and EMBASE using Pharmacotherapy, patient care, pharmaceutical care, pharmacist, and outcomes as search terms. Additional words were added where necessary to get the results from the databases. Inclusion criteria were articles published in English language, papers within the domain of the objective, articles with clearly defined methods, officially published documents, and unbiased articles. Duplicate articles and those without verifiable proofs were excluded. Out of 78 articles obtained for the study, 37 were excluded based on the selection criteria while 41 were used for the review.

\section{Discussion}

\subsection{Pharmaceutical Care Process}

PC is a cooperative, patient-centered system for achieving specific positive patient outcomes from the responsible provision of medicines. To achieve the outcomes, pharmacists cooperate with patients and with the other members of the healthcare team aimed at preventing or resolving drug therapy problems (DTP). The intention of prescribing drugs to a patient is treatment, prophylaxis, or diagnosis of medical conditions; however, these drugs may have negative effects on patients if not used appropriately or when they interact with other drugs. Pharmacists identify drug therapy problems (DTPs), resolve actual DTPs, and prevent potential DTPs through pharmaceutical care practices. A DTP is an undesirable event and/or risk experienced by a patient, which is associated with the use of drugs. The occurrence of a DTP could prevent or delay patients from achieving desired therapeutic goals. An actual DTP is an event that has already occurred in a patient, whereas a potential DTP is an event that is likely to develop if pharmacists do not make any appropriate interventions. DTPs pose significant challenges to health care providers and may affect morbidity and mortality. Drug therapy problems (DTPs) pose are a significant challenges to health care providers that are associated with morbidity, mortality, and patient's quality of life (QoL). Patients with type 2 diabetes mellitus receive a wide range of pharmacotherapeutic agents and are therefore at higher risk to experience $\mathrm{DTP}^{4-6}$.

Assessments establish full medication history and highlight actual and potential drug-related problems. Care plan states the goals to optimize care and the responsibilities of both the pharmacist and the patient in attaining the stated goals. Evaluation involves reviewing progress against the stated patient outcomes. The practice of clinical pharmacy is an essential component in the delivery of pharmaceutical care ${ }^{7}$. Pharmaceutical care has been shown to improve the outcomes of drug therapy in many disease conditions, including HIV/AIDS. The positive impact of pharmaceutical care interventions on the occurrence and resolution of drug therapy problems in antiretroviral therapy has been documented ${ }^{4-7}$.

\subsection{Perspective of Pharmaceutical Care in Some Selected Regions}

The historical philosophy of human care has always been a contentious subject across human needs, specialties, and basic interests. Right from the Age of Innocence to the modern times, care remains a conscious activity of human survivability and conviviality. Man at all times and circumstances require attention and tender loving care not only as prima facie health need, but also as part of the basic toning of psychosomatic balance. Pharmaceutical care as such is perceived as an intrinsic antecedent of an ever evolving and reforming the healthcare system and is as such understood as old in the new healthcare civilization. It therefore generally involves all ways and practices of providing service to patients and pharmaceutical practice via improved and standardized operating procedure to achieve patients-pharmacists satisfaction ${ }^{8}$.

In recent times, there has been a focus on patientcentered care rather than product-oriented care in pharmacy practice world over. This is because of the desire of pharmacists to meet the drug needs of the society through the carrying out of professional service. Numerous studies have demonstrated the inextricable relationship between morbidity-mortality and pharmacotherapy. Pharmaceutical care is a global human factor and public health philosophy still evolving around the world. However, PC apparently existed across human ages at different levels and practice, its 
modern definition, practice, and ethics provided the value latitude for pharmacists, pharmaceutical practice to function more expeditiously, tactfully, and professionally filling the widening gap between morbidity-mortality and pharmacotherapy. It is widely applied in various pharmaceutical settings in the United States. As at 2002, thousands of pharmaceutical care encounters have been documented with resultant cost savings ranging from 2:1 to $10: 1^{4}$. However, there are many debates as regards the different definitions ascribed to pharmaceutical care in various countries. These notional differences are results of differences in health care systems, resources, training, and educational requirements in various countries. The United Kingdom, the Netherlands, Sweden, Portugal and Nordic countries had a long tradition of pharmaceutical care while in other European countries like the Central and Eastern European countries, the practice is not as developed as in Western Europe ${ }^{9}$.Pharmaceutical care is a philosophy and practice in which the patient is the main beneficiary of the pharmacist's efforts. The pharmacist focuses his attitude, behavior, commitment, ideas, knowledge, responsibilities, and skills on the provision of drug therapy $y^{3,10}$.

\subsection{Procedures for Providing Patient- Pharmaceutical Care}

The steps in providing pharmaceutical care include establishing a professional/therapeutic relationship: the pharmacist should welcome and empathize with the patient. This will enable the patient to open up to the pharmacist. It is followed by a collection of patient-specific subjective and objective data: sources of patient data include patient interview, interview of other caregivers and patients' relatives, medical records review, laboratory report, and physical assessment. Evaluation of patients' data and identification of health, and drug therapy problems using critical thinking and problem solving skills, which helps to carry out the evaluation process, is an integral aspect of PC. This is followed by developing and implements pharmaceutical care plans. The written plan should indicate patient specific goals. Goals should be definite, achievable, measurable, and consistent with professional responsibilities. The pharmacist evaluates the interventions and follow-up, while assessing whether the interventions improved patient outcomes. It helps the pharmacist to know whether the management plan should be adjusted. The final stage is documentation and follow-up. This involves the recording of all activities that have been undertaken. It helps in proper follow up, and audit trail of patients. It is also a way of encouraging the generation of data needed for research and development in pharmaceutical care and related areas ${ }^{3}$.

\subsection{Education and Practice Interface}

Transition to PC provides was initiated by the Advanced Clinical Pharmacy Experience (ACPE) accreditation standards. Many Pharm. D graduates feel disillusioned when they attempt to join the workforce due to the disparity between their educational training and job specifications ${ }^{11}$.

\subsection{Benefits of Advanced Pharmacy Practice Experience (APPE)}

There are many beneficial aspects to developing an APPE and precepting students in the community practice setting. The benefits go beyond the educational benefits to students and extend to the profession, preceptors, sites, and the patients served ${ }^{12,13}$. The benefits to services, improve revenue for the service providers ${ }^{14}$.APPEs offer additional advantages for patients and the public. Having students provide in-depth care to patients will enable them to detect and avoid medication-related problems ${ }^{15,16}$. It helps students to carryout public and community health activities $^{17}$.

\subsection{Pharmaceutical Care as a Generalist Practice}

PC is applicable to the generality of population irrespective of their age or gender ${ }^{4}$.Other health professionals (e.g. physicians, nurses) also have clearly defined roles related to proper medication use. PC does not imply a reduction or elimination of those roles. In fact, PC is applicable to all areas of pharmacy practice that attend to patients ${ }^{3}$. Qualified health care providers other than pharmacists can provide pharmaceutical care. However, pharmacists possess knowledge and skills that make them uniquely suited. Pharmaceutical care practice shares three attributes common to all health care practices namely: a single philosophy of practice, one patient care process, and a practice management system ${ }^{18}$. 


\subsection{Philosophy of Pharmaceutical Care} Practice

PC explains what all practitioners should uphold. According to this philosophy, the practitioner performs the following:

- Takes responsibility for meeting society's need to reduce DTPs.

- Employs a patient-centered approach that addresses all the patient's drug-related needs.

- Establishes a caring therapeutic relationship with individual patients.

- Assumes a clearly defined set of responsibilities that directs patient care activities ${ }^{3,7}$.

\subsection{Patient Care Process}

The process of caring for patients explains the key processes involved in patient care and the duties of the practitioner in the practice. It involves patient review, formulation of the care plan, a continuation of care and noting down of all activities carried out. The activities are shown in Figure 1.

The pharmacists' pharmacotherapy workup is a tool available to practitioners that serve as a guide through the steps of the patient care process. It offers a standardized format for efficient documentation of patient-specific information needed for a financially viable pharmaceutical care practice ${ }^{20}$.

\subsection{Patient Assessment}

Patients are continually reviewed to ensure that they get optimum benefit from their medication therapy while minimizing DTP. DTP is any undesirable event involving drug therapy, which can affect the desired patient outcome. Seven major types of drug-related problems have been identified that relate to the appropriateness, effectiveness, safety, and convenience of drug therapy. There are multiple possible causes of each type of problem. Drug therapy problems drive subsequent steps in the patient care process; therefore, they are clearly stated and with a high level of specificity. Moreover, they are prioritized according to their degree of urgency as determined by the severity of potential harm to the patient ${ }^{21}$. Many relationships exist between drug-related needs and DTP ${ }^{22}$.

\subsection{Pharmaceutical Care Plan}

PC plan is targeted at eliminating every form of DTP or preventing them from occurring in the first place $^{23}$. It involves collaborations between patients and clinicians. Follow-up evaluation is essential in PC plan and process. The following processes are involved in follow-up evaluation process: determination of the actual outcomes that were achieved by the plan and assessment of the extent to which the plan has achieved the desired outcomes. Others are to determine if there are new or changing drug therapy problems that must be addressed and exploration of the factors predisposing patients to DTPs ${ }^{23,24}$.

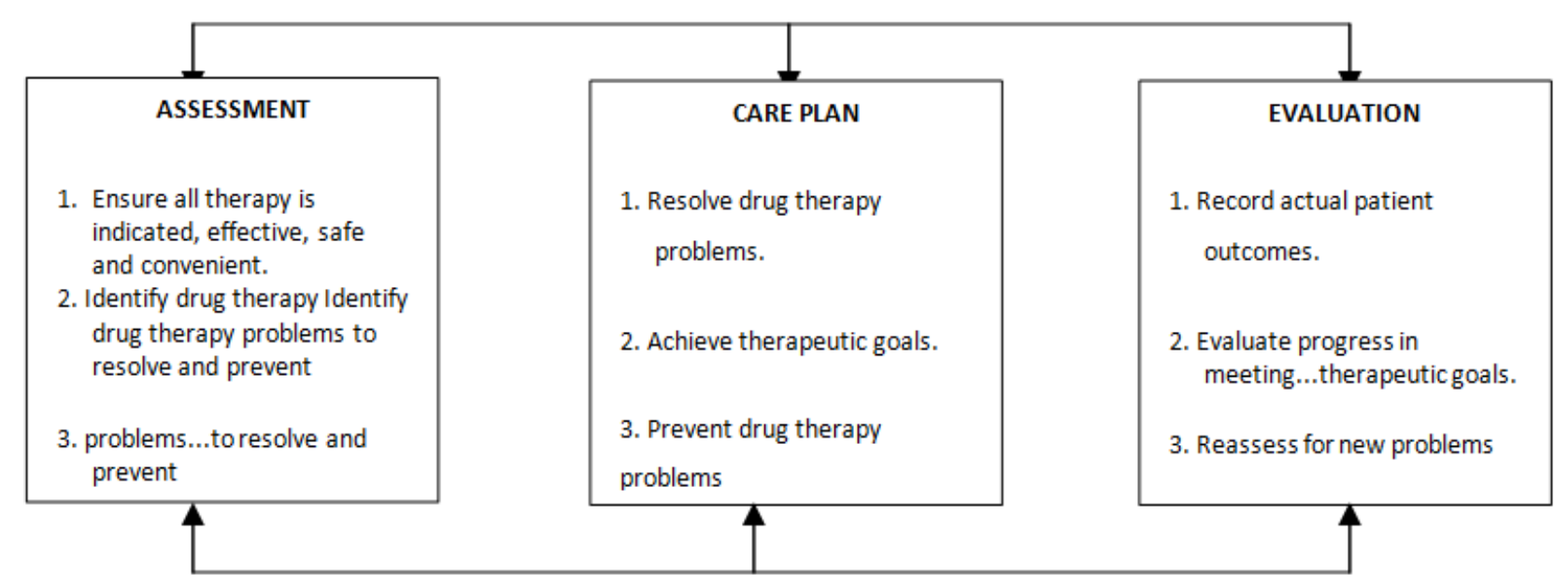

\section{Perform Continuous Follow Up}

Figure 1. The patient care process within a pharmaceutical care practice (Adapted from Cipolle et al 1998) ${ }^{19}$. (ie section 3.8). 
The status of each medical condition can be described according to the following categories:

- Resolved: the goals have been achieved and therapy is completed.

- Stable: the goals have been achieved, but continue the same therapy.

- Improved:progress is being made toward achievement of the goals, so continue the same therapy.

- Partial improvement: progress is being made, but minor adjustments in the therapy are required.

- Unimproved: there is no measurable progress yet, but continue the same therapy.

- Worsened: there is a decline in health, so revise the therapy accordingly.

- Failure: the goals are not achievable with the present therapy, so initiate new therapy.

- Expired: the patient died while receiving drug therapy ${ }^{19}$.

\subsection{Steps Involved in PC Process}

PC plan is individualized patient care plan with comprehensive medication therapy. It is necessary to carry the patient and physicians along in the process to ensure that a common goal is maintained and all the provided care documented. The steps in pharmaceutical care process are as follows:

Pharmacist establishes a professional relationship with the patient

- Greet the patient and introduce yourself

- Address patient by name or appropriate title in a respectful manner

- Know how the patient feels

- Show empathy

- Apply good communication skills and let the patient know that you are concerned and care about his/her health

- Introduce PC and its benefit to the patient

- Document the interaction properly

Collect patient's specific data by interviewing the patient and review of patients case records

- Collection of patients demographics

- Collection of previous medication history

- Document patient specific data

Evaluate patient drug therapy using collected data

- To know if the current medication is effective

- Assess if the conditions are being treated
- Determine the need for extra drugs

- Identify the potential and actual drug therapy problems

- Determine if any of the DTPs may have been caused by medications

- Document the DTPs in the PC daily worksheet and PC case notes for the information and action of other health care team members

\section{Develop PC plan}

- $\quad$ Prioritize the DTPs

- Determine the desired outcomes- clinical, economic and humanistic

- Develop definite, realistic and measurable patientfocused intervention (e.g. patient education, promote adherence, alleviate symptoms and prevent ADRs) and drug focused interventions e.g. substitution or switches, introducing a new drug to treat ADRs, control medication costs

- Specify monitoring parameters for DTPs and frequency of monitoring so that patients progress can be followed up e.g. laboratory test, clinical measurements, potential ADR, quality of life and patient satisfaction measurements and determine desired end points for each monitoring parameters and the frequency of monitoring

- Document the care plans in the PC daily information and action of other health team members

Implementation cares plan

- Implement the intervention according to the care plan and follow up

- Document the interventions in the PC daily worksheet and PC case notes for the information and action of other health care team members

Post-intervention evaluation and follow-up

- Evaluate the interventions using the pre-determined monitoring parameters for outcomes

- Document the outcomes in the PC daily worksheet and $\mathrm{PC}$ case notes for the information and action of other health care team members

- Use the data at this stage to provide feedback for the change or modification of the PC plan ${ }^{25}$

\subsection{Pharmaceutical Care Outcomes}

The relative importance of pharmaceuticals in health care is growing in most industrialized nations, as the percentage 
of health care expenditures for pharmaceuticals continues to increase. Spending on prescription drugs in the United States is increasing at a rate of $12 \%$ annually and will continue to increase approximately $10 \%$ per year over the next 8 years $^{26}$. PC outcomes may not be favorable always ${ }^{27}$. Huge resources are lost annually to drug therapy problems (DTP) related morbidity and mortality ${ }^{28}$.

DTPs cause many emergency room visits and admissions and most of them are preventable $e^{29,30}$. Preventable adverse outcomes related to drug use-often referred to as preventable drug-related morbidities (PDRMs) are typically not due to the inherent pharmacological properties of medicines, but rather to problems such as a lack of systematic approach to monitoring of patients receiving drug therapy by health professionals. A Canadian study estimated that the annual cost of PDRM in older adults in that country is $\$ 10.9$ billion (Canadian) CND. There are three main types of health-related outcomes should be considered: economic (drug-related and non drug-related), clinical (clinical events, physiologic and metabolic measures), and humanistic outcomes such as patient satisfaction ${ }^{31,32}$.

Perceived satisfaction with medical treatment is vital to determining the overall well-being and health of patients and is recognized as being one of the main components of quality. Health-related quality of life (HRQoL) relates to those aspects of a patient's life dominated by personal health. Although differing opinions exist, there is some consensus that there are four main dimensions and a social dimension. Increasingly, HRQoL is a humanistic outcome measure used in clinical drug trials and in pharmacy program evaluations ${ }^{16}$. PC outcomes need to be evaluated to ensure that process is effectiveness. Wrong use of medications predisposes to poor outcomes in patients ${ }^{33}$.Patient care quality measurements are a challenging process in health care delivery. However, it is very necessary ${ }^{34}$.

\subsection{Roles of Various Stakeholders in Pharmaceutical Care Outcomes Measurement}

Enhancing medication use outcomes is the duty of all stakeholders involved in health care delivery. The objectives of care should be clearly stated and must be specific, measureable, appropriate, and time bound ${ }^{2,3}$.
Measureable outcomes help in better and targeted patients care and follow up assessment.

\subsection{Patients' Quality of Life}

Patients' quality of life is affected by individual's physical well-being, psychological state, and level of independence. Social dealings, patients health status, disease state and relationship with the relevant features of their environment, are other factors that affect patients quality of life ${ }^{35}$.The Quality of life (QoL) could be thought of as a complex construct incorporating an individual's subjective judgment of physical, affective and social well being, including both a cognitive aspect (satisfaction) and an emotional aspect (happiness) ${ }^{36}$.QoL issues help to predict an individual's ability to deal with his ailment and maintain lasting health and well-being. Standardized generic instruments are now used in tracking patients QoL to evaluate their expectations, well-being, and satisfaction. Examples include SF-8, SF-12, and SF-36 ${ }^{4,36}$.

Various studies have shown that type 2 diabetes patients have a low quality of life, with older patients having a lower quality of life; especially pertaining to physical performance, roles restrictions due to physical, emotional and social functioning ${ }^{37,38}$. Females have a lower quality of life, possibly because of less involvement in physical activity and poorer social conditions ${ }^{38,39}$. Patients with co-morbidities have poor quality of life though some chronic conditions like cancer, cardiovascular diseases, pulmonary diseases, and diabetes mellitus are more strongly linked with poor quality of life than others. The most frequent co-morbidity is hypertension, while poly-neuropathy, dyslipidemia, ophthalmological complications may also be present ${ }^{37}$.

\subsection{Factors Affecting Quality of Life}

- Lower income/low rated employment: low income of patients limits their access to medical interventions such as drugs, which might be relatively expensive to procure.

- Lower educational status: patients with low educational background tend to have a lower quality of life. This could be because of little or no knowledge with respect to the disease, its risk factors, complications, and management.

- Age: the incidence of most chronic disease diseases 
like diabetes, and hypertension increases with increase in age leading to declining in quality of life.

- Body mass index: patients with body mass index greater than $30 \mathrm{~kg} / \mathrm{m}^{2}$ classified as obese patients are more prone to develop insulin resistance, which predispose to T2DM.

- Sex of patient: females are easily affected by childbirth, poor social conditions and less involvement in physical activities which reduces their quality of life, unlike their male counterparts.

- Smoking status: smokers have a lower quality of life because they are more prone to respiratory tract diseases which reduce their immunity.

- Treatment type: there has been controversy as to whether a diabetic treatment regimen has an effect on a patient's quality of life. A study found that diabetic patients managed with insulin appear to be more stable concerning the quality of life. They are less prone to majority of disease-related factors (co-morbidity, weight gain among others) when compared to patients on oral hypoglycemic agents ${ }^{38-40}$.

Diabetes mellitus modifies a patient's life permanently. Patient's self-care consisting of oral anti-diabetes agents, daily insulin injections, self-monitoring of blood glucose level and diet has an effect on QoL. In a review, it was concluded that diabetes patients have worse quality of life than non-diabetic patients do, especially in the areas of physical performance and well-being. Complications of diabetes (microvascular and macrovascular) have a negative effect on quality of life $\mathrm{e}^{41}$. A study did show that diabetic complications have more effect on the quality of life in patients under 65 years. This may be as a result of less co-morbidity associated with these patients thus have not accustomed to the idea of accepting poor quality of health or due to responsibilities such as work and family not found in the older patients $s^{37,41}$.

\subsection{Assessment of Quality of Life}

The aim of assessing quality of life in diabetic patients is to have an objective evaluation of how and how much diabetes affects patients' life and how patients deal with it. Instruments are available for the measurement of emotional, physical, and social aspects of QoL for diabetic patients. These are generic and disease specific questionnaire. Generic instruments are appropriate for healthy people as well persons with diseases, thus enabling comparisons to be made between dead patients and the general population. Disease-specific instruments concentrate on a population with a particular disease and are sensitive to effects of treatment and changes over time than the former. An example of a generic instrument is the WHOQoL instrument, short version (WHOQoL-Bref). WHOQoL-Bref is a 26 -item generic questionnaire that emphasizes the subjective response of patients rather than objective conditions, developed in various languages ${ }^{35}$.

The WHOQoL is made up of domains (dimensions) and facets (sub domains). The Medical Outcome Study Health Survey (SF-36) is also a generic questionnaire that assesses six domains of functional health status through physical functioning, effect of physical illness on role functioning, perception of general health, effect of illness on social functioning, discomfort because of pain, and mental health ${ }^{37}$. An example of a disease-specific questionnaire is the Diabetes Quality of Life Measure (DQoL), with 46 core items rated by the respondent on a 5-point Likert-type scale ranging from 1 to 5 . A perfect instrument for the measurement of QoL in diabetes mellitus should integrate the benefits of both generic and diabetes-specific instruments, as well as be sensitive to changes, offer information about diabetes-specific associations with QoL, permit comparisons among diverse groups of patients or general populace and make economic evaluations possible ${ }^{35,37,41}$.

\section{Conclusion}

Pharmaceutical care is a patient cantered process, which utilizes pharmacist's expertise in a multidisciplinary and collaborative patient care process to improve patient's clinical, economic, and humanistic outcomes. It is a model of practice and a philosophy. Patient centeredness in PC is an approach that is generalist in nature because every patient is taken care of in a holistic and individualistic manner. It is a measure of quality with measureable indicators where the patients, clinicians and caregivers all have roles to play towards improved patient outcomes. The need for PC is invaluable especially in chronic disease management where compromise in management results in loss of resources, complications, disabilities, and death. Embracing this trend promptly will be invaluable to developing countries with high level of poverty and limited resource allocation to healthcare. 


\section{Conflict of Interest}

The authors have none-to declare.

\section{References}

1. Helper CD, Strand LM. Opportunities and responsibilities in pharmaceutical care. American Journal of Hospital Pharmacists.1990; 47:533-43.

2. American Society of Hospital Pharmacists. ASHP statement on pharmaceutical care. American Journal of Hospital Pharmacy.1993; 50:1720-30.

3. Hepler CD, Strand LM. Opportunities and responsibilities in pharmaceutical care. American Journal of Hospital Pharmacy.1990; 47:533-43.

4. Oparah AC. Pharmaceutical care concept, philosophy, competency, and benefits. In: Oparah AC, editor. Essentials of Pharmaceutical care. Lagos: Cybex Publication;2010; $1: 1-55$.

5. Webb DG, Davies JG, McRobbie D. Clinical Pharmacy process. In: Walker R, Whittle-sea C, editors. Clinical Pharmacy and Therapeutics. New York: Elsevier; 2012; 1:2-13.

6. Nwaozuzu EE, Okonta JM, Aguwa CN. Impact of pharmaceutical care interventions on the occurrence and resolution of drug therapy problems in antiretroviral drug therapy. International Journal of Development and Sustainability.2013; 2(1): 415-29.

7. Hepler CD, Strand LM. Opportunities and responsibilities in pharmaceutical care. American Journal Pharmacy Education. 1989; 1:75-145.

8. Farris KB, King D. Assessing the quality of pharmaceutical care I: One perspective of quality. Annals of Pharmacotherapy. 1993; 27:68-73, 215-23.

9. Berenguer B, la Casa C, de la Matta MJ, Martin-Calero MJ. Pharmaceutical Care: Past, Present, and Future. Current Pharmaceutical Discoveries. 2004; 16:3931-46.

10. Posey LM. Pharmaceutical Care: The Re-professionalization of Pharmacy. In: Posey LM, editor. Pharmacy Cadence 1992.PAS Pharmacy/Association Services: Athens, Georgia; 1992; 15-22.

11. Syracuse MV, Schondelmeyer SW, Hadsall RS. Accessing career aspiration of pharmacy students. American Journal of Pharmacy Education. 2004; 68: Article 75.

12. Scott DM, Narducci WA, Tungnickei PW. Pharmaceutical care preceptor training and assessment in community pharmacy clerkship sites. American Journal of Pharmacy Education. 1999; 63:265-71.

13. American Pharmaceutical Association Special Report (APhASR). Opportunities for the Community Pharmacist in Managed Care. Washington: American Pharmaceutical Association;1994.

14. Willink DP,Isetts BJ. Becoming indispensable: developing innovate community pharmacy practices. Journal of American Pharmacists Association.2005; 45:376-86.
15. Anderson RJ, Nykamp D, Miyahara R. Documentation of pharmaceutical care activities in community pharmacies by doctors of pharmacy students. Journal of Pharmacy Practice; $1995 ; 7(2): 83-8$.

16. Mackinnon GE. Analysis of pharmacy student interventions collected via an internet based system. American Journal of Pharmacy Education. 2003; 67: Article 90.

17. Calls KA, Hutchison LC, Elliot ME. Health People 2010: challenges, opportunities, and a call to action for America's pharmacists. Pharmacotherapy. 2004; 24:1241-94.

18. Al-shaqha WN, Zaira M. Pharmaceutical care management: a modern approach to providing seamless and integrated health care. International Journal of Health Care Quality Assessment. 2010; 1(1):22-4.

19. Cipolle RJ, Strand LM, Marley PC. Pharmaceutical care practice. New York:McGraw-Hills;1998. p. 1-124.

20. McCallian DJ, Carlstedl BD, Rupp MT. Elements of a pharmaceutical care plan. Journal of American Pharmaceutical Association. 1999; 39:82-3.

21. Wolf $\mathrm{HH}$, Walton CA, Hepler CO, Koda-Kimble MA, Knapp DA, Miller KW, Nahata MC Rutledge CO, Smith N. Change in Pharmaceutical education. A position paper. AACP News.1991 Nov; 1-13.

22. Longe RL, Calvert JC, Young LY. Physical assessment: aguide for evaluating drug therapy. Ann Arbor, MI: Applied TherapeuticsIncorporated.1994;2:1-12.

23. Smith WE, Benderev K. Levels of pharmaceutical care: a theoretical model. American Journal of Hospital Pharmacy. 1991; 48:540-6.

24. Enright SM. Assessing patient outcomes. American Journal of Hospital Pharmacy. 1988; 45(45): 1376-8.

25. Strand LM. Building a practice in pharmaceutical care. Pharmacy Journal.1998; (1):260, 874-8.

26. Mehl B, Santell TP. Projecting future drug expenditures 2001. American Journal of Health System Pharmacists.2001; 58:125-33.

27. Linda TK, Janet MC, Molla SD. To err is human: building a safer health system. Washington DC: National Academy Press. 1999.p. 1-34.

28. FR, Grizzle AJ. Drug-related morbidity and mortality: updating the cost of illness model. Journal of American Pharmaceutical Association. 2001; 41(2):192-9.

29. Grymonpre RE, Didur CD, Montgomery PR, Sitar DS. Pill count, self-report, and pharmacy claims data to measure medication adherence in the elderly. Annals of Pharmacotherapy. 1988 ; 32:749-54.

30. Nelson KM, Talbert RL. Drug-related hospital admissions. Pharmacotherapy. 1996; 16(4):701-7.

31. Kidney T, Mackinnon NJ. Preventable drug-related morbidity and mortality in older adults: A Canadian Cost-ofillness model. Geriatrics Today. 2001; 4(3):120.

32. Kozona CM, Reeder E, Schulz RM. Economic, Clinical, and humanistic outcomes: A planning model for pharmaco-economic research. Clinical Therapeutics. 1993; 15:1121-32. 
33. Hannan EL. The continuing quest for measuring and improving access to necessary care. Journal of American Medical Association.2006; 284(18):2374-6.

34. Fihn SD. The quest to quantify quality. Journal of American Medical Association. 2000; 283(13): 1740-2.

35. Issa BA, Baiyewu O. Quality Of Life of Patients with Diabetes mellitus in a Nigerian Teaching Hospital. Hong Kong Journal of Psychiatry.2006; 16(1):27-33.

36. Richard RR, Mark P. Quality of Life and Diabetes. Diabetes, metabolism, respiration and review. 1999; 15(3):205-18.

37. Ana S, Radmila V, Aleksandra CĐ, Nikola S, Tatjana C. Quality of life in type 2 diabetic patients. Scientific Journal of the Faculty of Medicine in New Zealand. 2014; 31(3):193-200.

38. Agu KA, Oqua D. Concept of Pharmaceutical Care in HIV/AIDS, In: Oqua D, Agu KA, Isah MA, et al., Editors.
GHAIN Pharmaceutical Care in HIV/AIDS Training for Hospital Pharmacists, Participants Manual. 2011;1-8.

39. Anumol M, Anusree TK, Aparna Mathew A,Archana S, Athira M, Sachina BT, Gireesh GR. Quality of life among type-II Diabetes mellitus patients in south India: A Descriptive Study. American International Journal of Research in Humanities, Arts, and Social Sciences. 2014; 7(2):197-200.

40. Andrzej MF, Beata J, Izabella U, Mariola S, Bernard P, Jacek P. Type 2 diabetes quality of life patients treated with insulin and oral hypoglycemic medication. ActaDiabetologica.2011; 48:237-42.

41. UK Prospective Diabetes Study Group. Quality of life in type 2 diabetic patients is affected by complications but not by intensive policies to improve blood glucose or blood pressure control (UKPDS 37). Diabetes Care.1999; 22(7):1125-36. 